\title{
Morbilidad y mortalidad por accidentes de tránsito según componentes temporales, Ecuador
}

\section{Morbidity and mortality due to traffic accidents according to temporary components, Ecuador}

\author{
Antonio R. Gómez García ${ }^{1 *}$, Viviana Orbe Montenegro ${ }^{2}$ y Yolis Y. Campos Villalta ${ }^{2}$ \\ ${ }^{1}$ Universidad Espíritu Santo \\ ${ }^{2}$ Universidad Internacional SEK, Ecuador \\ *agomezg@uees.edu.ec
}

DOI: https://doi.org/10.26871/killkana_salud.v3i1.246

\begin{abstract}
Resumen
Introducción: en Ecuador los accidentes de tránsito son considerados como un problema de salud pública, por lo que ocupan los primeros puestos en tasa de fallecimientos a nivel mundial. Objetivo: describir el componente temporal como factor incidente de la morbilidad y mortalidad por accidentes de tránsito en el año 2016. Material - Métodos: análisis descriptivo de corte transversal con representación temporal de los siniestros, víctimas con lesión y personas fallecidas a través de datos estadísticos oficiales. Se calcularon estadísticos descriptivos, tasas de morbilidad y mortalidad, Coeficiente de Correlación de Pearson $(\mathrm{p}<0.05 ; \mathrm{p}<0.01)$ y Riesgo Relativo (RR; IC95\%). Resultados: se registraron un total de $30.269(2.522 \pm 233)$ siniestros, 21.388 víctimas con lesión $(1.782 \pm 150)$ y $1.967(164 \pm 16)$ personas fallecidas por accidentes de tránsito Las tasas de morbilidad mensual presentan estacionalidad a diferencia de la mortalidad $(\mathrm{p}<0.05)$ : junio $(71,6 \times 1.000)$, octubre $(74,7 \times 1.000)$ y noviembre $(71,3 \times 1.000)$. Los días sábados y domingos la probablidad de sufrir lesiones (RR: 1,05; 1C95\%: 1,03-1,07) y fallecer (RR: 1,46; 1C95\%: 1,34-1,59) es mayor respecto de lunes a viernes. En especial, en horario nocturno a partir de las 20:00 h. $(\mathrm{p}<0.05)$. Conclusiones: los accidentes de tránsito siguen un patrón temporal de agrupamiento a largo de todo el año. Los resultados hallados constituyen una valiosa información para establecer estrategias y campañas puntuales en cuanto al mes, día y franjas horarias. Es necesario continuar con estudios que incluyan períodos más amplios para conocer los cambios en los patrones temporales de los accidentes de tránsito, promover una cultura de seguridad vial y peatonal en la sociedad ecuatoriana y mejorar los sistema estadística en el país.
\end{abstract}

Palabras clave: accidentes de transito; morbilidad; mortalidad; Ecuador.(DeCS).

\begin{abstract}
Introduction: in Ecuador, road traffic accidents are considered a public health problem and are among the highest death rates in the world. Objective: To describe the temporal component as an incidental factor in road traffic morbidity and mortality in 2016. Material - Methods: descriptive cross-sectional analysis with a temporal representation of accidents, injured victims and deceased persons by using official statistical data. Descriptive statistics, morbidity and mortality rates, Pearson's Correlation Coefficient $(p<0.05 ; p<0.01)$ and Relative Risk $(R R ; 95 \%$ CI) were calculated. Results: out of a total of 30,269 (2,522 \pm 233$)$ registered accidents, 21,388 resulted in injured victims (1,782 \pm 150$)$ and 1,967 (164 \pm 16)in deceased persons. Monthly morbidity rates present invariability, as opposed to mortality ( $p<0.05)$ : June (71.6 $x$ 1.000), October $(74.7 \times 1.000)$, and November $(71.3 \times 1,000)$. The probability of suffering injuries (RR: 1.05 ; lC95\%: 1.03-1.07) and dying (RR: 1.46; lC95 \%: 1.34-1.59) on Saturdays and Sundays, is higher than on Monday to Friday. Especially, at night times, after 8:00 p.m. (p<0.05). Conclusions: traffic accidents follow a temporal clustering pattern throughout the year. The results found constitute valuable information for the establishment of strategies and campaigns which are specific to months, days and time zones. It is necessary to continue with these studies over longer periods of time to find changes in the temporal patterns of traffic accidents, to promote a road and pedestrian safety culture in Ecuadorian society, and to improve the statistical systems in the country.
\end{abstract}

Key words: accidents, traffic; morbidity; mortality; Ecuador. (MeSH). 


\section{Introducción}

Los accidentes de tránsito (A.T.) constituyen una de las principales causas de morbilidad y mortalidad en todo el mundo. ${ }^{1}$ Ecuador ocupa el séptimo puesto en Latinoamérica en tasa de fallecimientos por esta causa y el décimo tercero a escala global en comparación con países de ingresos similares, ${ }^{2}$ debido al crecimiento demográfico y aumento del parque vehicular en las últimas décadas. ${ }^{3}$

A pesar de las acciones públicas en el país, en la actualidad los A.T. son considerados como un problema de salud pública y han sido estudiados recientemente desde diferentes enfoques epidemiológicos ${ }^{4}$ y espaciales. ${ }^{5,6}$

En particular, la población joven de las principales provincias ecuatorianas son las más vulnerables, generando importantes pérdida en años de vida ${ }^{7}$ y suscitando la necesidad prioritaria de establecer acciones para mejorar la seguridad vial a nivel nacional. Sin embargo, a través de la bibliografía consultada, el componente temporal sobre de los A.T. aún no han sido suficientemente estudiados hasta el momento. ${ }^{4}$

El objetivo de este estudio es describir el comportamiento del componente temporal como factor incidente en la morbilidad y mortalidad por A.T. en el Ecuador. El conocimiento de este análisis constituye una valiosa información y permite establecer programas y campañas puntuales en seguridad vial. ${ }^{8}$

\section{Materiales y Métodos}

Se realizó un estudio descriptivo de corte transversal con representación temporal de los A.T. registrados en el año 2016 a través de los datos estadísticos disponibles en la Agencia Nacional de Tránsito, ${ }^{9}$ Ecuador.

Las variables de análisis fueron los siniestros, víctimas con lesión y personas fallecidas en A.T., distribuidos a partir de componentes temporales: ${ }^{3}$ (i) mes, (ii) día de la semana y (iii) franja horaria de ocurrencia de los accidentes.

A partir de esta información, se calcularon las tasas de morbilidad y mortalidad: ${ }^{10}$

Tasas de Morbilidad $=\frac{(L)}{A T} \times 100$

- L: número víctimas con lesión.

- $\quad$ AT: número de accidentes de tránsito.

Tasas de Mortalidad $=\frac{(M)}{A T} \times 1,000$

- M: número de fallecidos

- AT: número de accidentes de tránsito.

Luego, se utilizó el Coeficiente de Correlación de Pearson $(\mathrm{p}<0.05 ; \mathrm{p}<0.01)$ para conocer la asociación estadística lineal y la estimación de probabilidad de ocurrencia temporal de los A.T. mediante el Riesgo Relativo (RR) con sus respectivos intervalos de confianza (IC95\%). Asimismo, se presentan las frecuencias absolutas y relativas, medias, desviación estándar y porcentajes de variación.

Se empleó el software Statistical Package for the Social Sciences (SPSS, versión 24.0) para el análisis estadístico.

Tabla 1. Distribución mensual y semanal de los siniestros, lesionados y fallecidos por A.T. en 2016, Ecuador

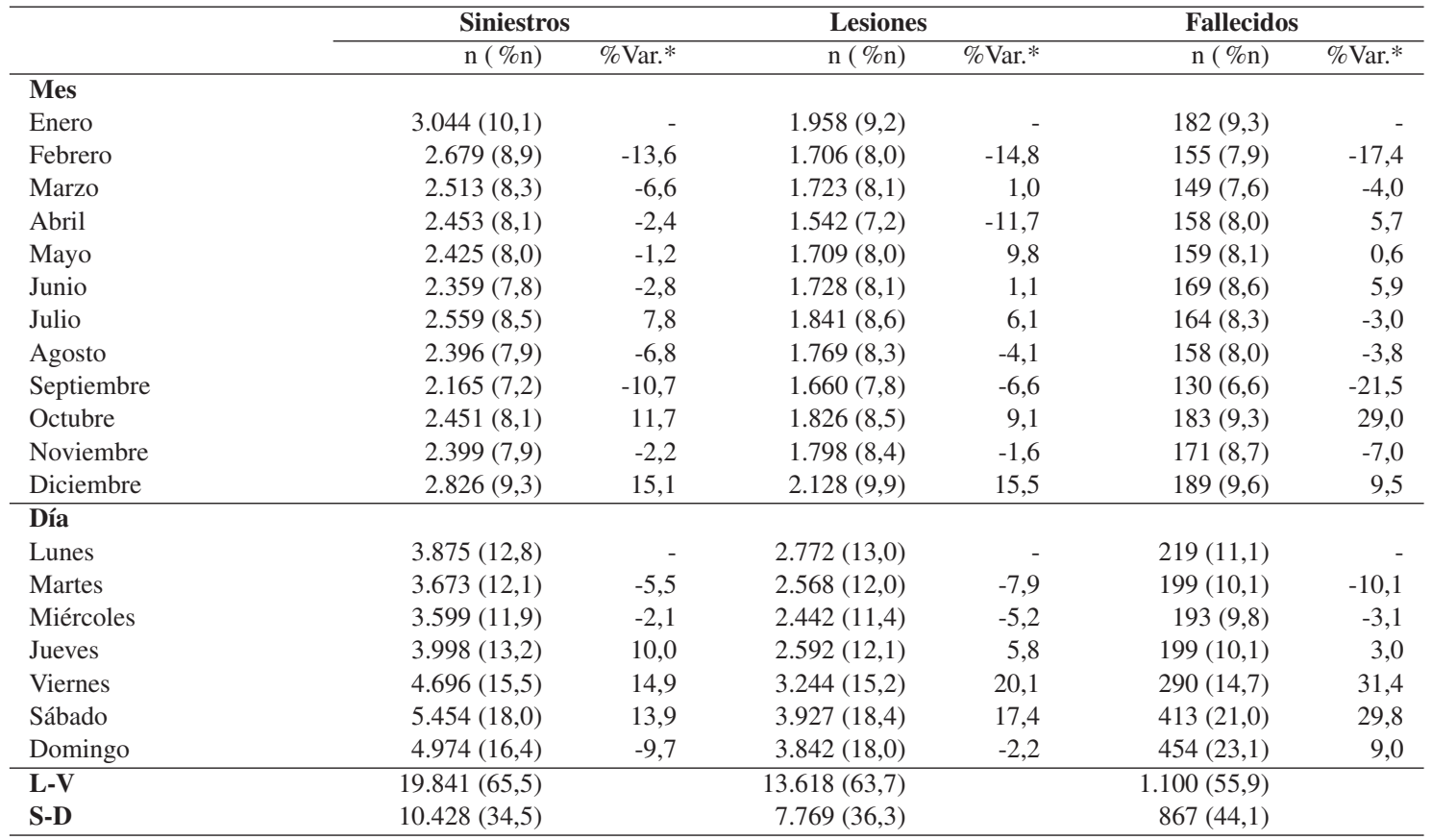

* Porcentaje de Variación respecto al mes y/o día anterior 


\section{Resultados}

Durante el año 2016 se registraron un total de 30.269 de siniestros por A.T. con una media de $2.522 \pm 233$ mensual. Se observa que enero es el mes con mayor accidentalidad seguido de diciembre, Tabla 1. A partir del mes de febrero comienza un descenso progresivo del número de siniestros hasta la mitad de año.

En cuanto a las víctimas con lesión, los A.T. originaron 21.388 lesionados con una media de $1.782 \pm 150$ cada mes, fluctuando el número mensual entre 1.542 víctimas en abril a 2.128 en diciembre. Finalmente, se registraron un total de 1.967 personas fallecidas, con una media de $164 \pm 16$ mensual, diciembre es el mes con mayor número de eventos para ambos casos.

Se observa una asociación estadísticamente significativa entre los siniestros, víctimas con lesiones y fallecidos por A.T. $(\mathrm{p}<0.05)$ en los meses de estudio analizados y con mayor fuerza de asociación $(\mathrm{p}<0.01)$ entre lesionados $y$ fallecidos.

En función a la ocurrencia de los A.T. por día de la semana (Tabla 1), los sábados y domingos corresponden a los días con mayor número de eventos en siniestros, lesionados y fallecidos, conforme se aproxima el fin de semana se observa una tendencia al aumento y asociación estadística $(\mathrm{p}<0.01)$. Destaca el miércoles como el día de la semana con menor porcentaje de eventos por A.T.

En la Tabla 2 se presenta la distribución de los siniestros, lesionados y fallecidos por A.T. según franja horaria. El mayor número eventos por siniestros se presenta entre las 06:00 y 22:59 horas, siendo el punto máximo entre las 19:00 A 19:59 horas.

Asimismo, entre las 13:00 y 20:59 horas es cuando mayor número de víctimas con lesión se registraron por A.T., destaca igualmente con 1.100 víctimas la franja horaria entre las 07:00 y 07:59 horas.

Los fallecimientos por accidentes de tránsito se concentran principalmente entre las 19:00 y 20:59 horas con 260 personas fallecidas, entre las 05:00 y 06:59 horas con 223 y con 103 fallecidos entre las 16:00 - 16:59 h. Se observa una asociación estadísticamente significativa $(\mathrm{p}<0.05)$ entre el horario diurno (08:00 - 19:59 h.) y nocturno (20:00 - 07:59 h.)

Tabla 2. Distribución horaria de los siniestros, lesionados y fallecidos por A.T. en 2016, Ecuador

\begin{tabular}{|c|c|c|c|c|c|c|}
\hline & \multicolumn{2}{|c|}{ Siniestros } & \multicolumn{2}{|c|}{ Lesiones } & \multicolumn{2}{|c|}{ Fallecidos } \\
\hline & $\mathrm{n}(\% \mathrm{n})$ & $\%$ Var. $^{*}$ & $\mathrm{n}(\% \mathrm{n})$ & \%Var.* & $\mathrm{n}(\% \mathrm{n})$ & \% Var.* \\
\hline \multicolumn{7}{|l|}{ Hora } \\
\hline 00:00 - 00:59 & $892(2,9)$ & - & $527(2,5)$ & - & $74(3,8)$ & - \\
\hline $01: 00-01: 59$ & $773(2,6)$ & $-15,4$ & $546(2,6)$ & 3,6 & $74(3,8)$ & 0,0 \\
\hline $02: 00-02: 59$ & $771(2,5)$ & $-0,3$ & $439(2,1)$ & $-24,3$ & $63(3,2)$ & $-17,5$ \\
\hline $03: 00-03: 59$ & $718(2,4)$ & $-7,4$ & $483(2,3)$ & 9,1 & $56(2,8)$ & $-12,5$ \\
\hline $04: 00-04: 59$ & $642(2,1)$ & $-11,8$ & $381(1,8)$ & $-26,7$ & $76(3,9)$ & 26,3 \\
\hline $05: 00-05: 59$ & $870(2,9)$ & 26,2 & $570(2,7)$ & 33,1 & $114(5,8)$ & 33,3 \\
\hline $06: 00-06: 59$ & $1.298(4,3)$ & 33,0 & $943(4,4)$ & 39,5 & $109(5,5)$ & $-4,6$ \\
\hline $07: 00-07: 59$ & $1.584(5,2)$ & 18,1 & $1.100(5,1)$ & 14,3 & $83(4,2)$ & $-31,3$ \\
\hline $08: 00-08: 59$ & $1.356(4,5)$ & $-16,8$ & $941(4,4)$ & $-16,8$ & $69(3,5)$ & $-20,3$ \\
\hline 09:00 - 09:59 & $1.225(4,0)$ & $-10,7$ & $771(3,6)$ & $-22,1$ & $61(3,1)$ & $-13,1$ \\
\hline $10: 00-10: 59$ & $1.335(4,4)$ & 8,2 & $983(4,6)$ & 21,6 & $76(3,9)$ & 19,7 \\
\hline $11: 00-11: 59$ & $1.308(4,3)$ & $-2,1$ & $994(4,6)$ & 1,0 & $70(3,6)$ & $-8,6$ \\
\hline $12: 00-12: 59$ & $1.439(4,8)$ & 9,1 & $984(4,6)$ & $-1,0$ & $61(3,1)$ & $-14,8$ \\
\hline $13: 00-13: 59$ & $1.526(5,0)$ & 5,7 & $1.186(5,5)$ & 17 & $78(4,0)$ & 21,8 \\
\hline $14: 00-14: 59$ & $1.424(4,7)$ & $-7,2$ & $1.060(5,0)$ & $-11,8$ & $76(3,9)$ & $-2,6$ \\
\hline $15: 00-15: 59$ & $1.567(5,2)$ & 9,1 & $1.043(4,9)$ & $-1,7$ & $63(3,2)$ & $-20,6$ \\
\hline $16: 00-16: 59$ & $1.602(5,3)$ & 2,2 & $1.129(5,3)$ & 7,7 & $103(5,2)$ & 38,8 \\
\hline $17: 00-17: 59$ & $1.617(5,3)$ & 0,9 & $1.220(5,7)$ & 7,4 & $80(4,1)$ & $-28,8$ \\
\hline $18: 00-18: 59$ & $1.591(5,3)$ & $-1,6$ & $1.207(5,6)$ & $-1,1$ & $79(4,0)$ & $-1,3$ \\
\hline $19: 00-19: 59$ & $1.711(5,7)$ & 7,0 & $1.270(5,9)$ & 5,0 & $145(7,4)$ & 45,5 \\
\hline $20: 00-20: 59$ & $1.605(5,3)$ & $-6,6$ & $1.158(5,4)$ & $-9,7$ & $115(5,8)$ & $-26,1$ \\
\hline $21: 00-21: 59$ & $1.312(4,3)$ & $-22,3$ & $955(4,5)$ & $-21,2$ & $81(4,1)$ & $-42,0$ \\
\hline $22: 00-22: 59$ & $1.145(3,8)$ & $-14,6$ & $803(3,8)$ & $-18,9$ & $77(3,9)$ & $-5,2$ \\
\hline $23: 00-23: 59$ & $958(3,2)$ & $-19,5$ & $694(3,2)$ & $-15,8$ & $84(4,3)$ & 8,3 \\
\hline 08:00 - 19:59 & $17.701(58,5)$ & & $12.787(59,8)$ & & $961(48,9)$ & \\
\hline 20:00 - 07:59 & $12.568(41,5)$ & & $8.600(40,2)$ & & $1.006(51,1)$ & \\
\hline
\end{tabular}

La tasa de morbilidad mensual presenta estacionalidad, observándose diferencias estadísticas significativas, destaca septiembre como el mes de mayor morbilidad; por cada 100 siniestros por A.T. se originaron 78 víctimas con lesión (Figura 1).
En lo que respecta a la tasa de mortalidad durante el año 2016, se observan variaciones mensuales, en particular, junio $(71,6 \times 1.000)$, octubre $(74,7 \times 1.000)$ y noviembre $(71,3 \times 1.000)$ son los meses que presentan mayor tasa de mortalidad $(\mathrm{p}<0.05)$. 
La tasa de morbilidad por A.T. según el día muestran una tendencia al aumento conforme se aproxima el fin de semana a lo largo del año (Figura 2), los domingos por cada 100 accidentes ocasionaron 77 víctimas con lesión frente a 65 víctimas los días jueves.

Con mayor tendencia al aumento y asociación ( $\mathrm{p}<0,01)$, la tasa de mortalidad se incrementa en los fines de semana; de lunes a viernes por cada 1.000 accidentes de tránsito fallecieron 55 personas frente a las 83 entre los sábados y domingos. El jueves, es el día de menor tasa de morbilidad y mortalidad.

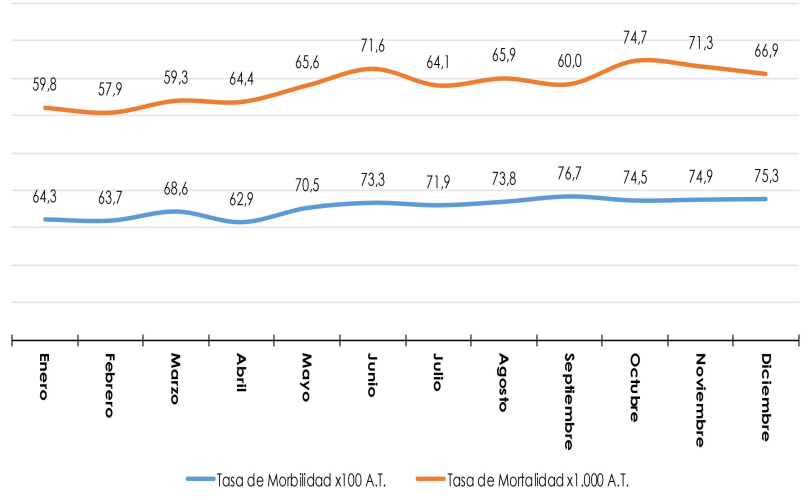

Fig. 1. Tasas de morbilidad y mortalidad por A.T. según mes. en 2016, Ecuador

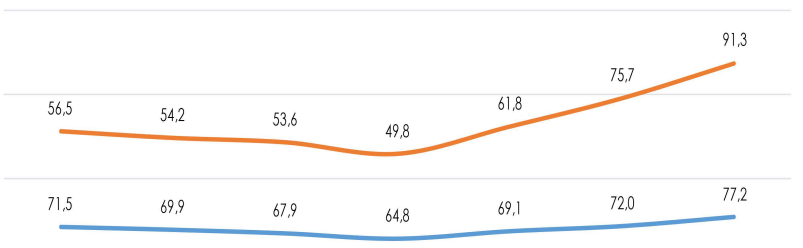

\begin{tabular}{|c|c|c|c|c|c|c|}
\hline 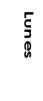 & 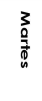 & $\begin{array}{l}3 \\
\frac{3}{0} \\
\frac{0}{0} \\
\frac{0}{0} \\
\omega\end{array}$ & 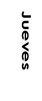 & 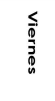 & $\begin{array}{l}u \\
0 \\
0 \\
0 \\
0 \\
0\end{array}$ & $\begin{array}{l}0 \\
0 \\
3 \\
\frac{3}{3} \\
0\end{array}$ \\
\hline
\end{tabular}

Fig. 2. Tasas de morbilidad y mortalidad por A.T. según día de la semana. en 2016, Ecuador

Para el análisis de las tasas morbilidad y mortalidad por A.T. según la hora, se dividió el día en dos franjas como se muestra en la Figura 3 (A-B).

La franja horaria de 08:00 - 19:59 presenta la mayor tasa de morbilidad (72 x 100), inversamente proporcional a lo observado en la franja horaria de 20:00 - 07:59 que registra la mayor tasa mortalidad $(80 \times 1.000)$ en accidentes de tránsito.

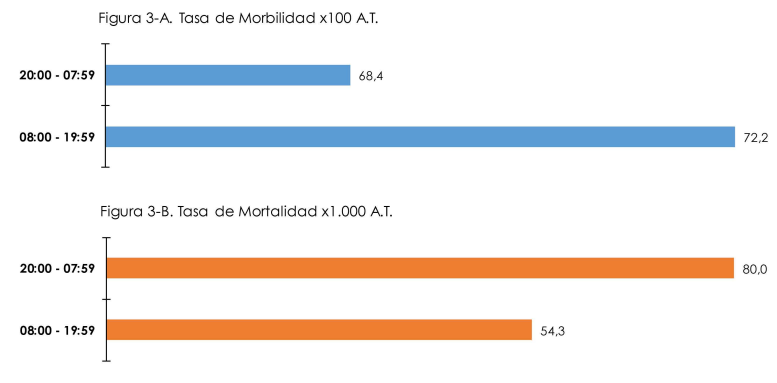

Fig. 3. (A-B). Tasas de morbilidad y mortalidad por A.T. según franja horaria en 2016, Ecuador

En cuanto a la probabilidad de sufrir lesiones por A.T. en 2016, octubre (RR: 1,10; 1C95\%: 1,06-1,14), septiembre (RR: 1,05; 1C95\%: 1,01-1,09) y diciembre (RR: 1,04; 1C95\%: 1,01-1,08) son los meses del año donde existe mayor riesgo de sufrir víctimas con lesión, asimismo, en los meses de octubre (RR: 1,15; 1C95\%: 1,00-1,34), junio (RR: 1,10; 1C95\%: 0,95-1,29) y noviembre (RR: 1,10 ; 1C95\%: 0,94-1,28) existe mayor riesgo de fallecer por esta causa.

En función a la distribución semanal, en los días sábados y domingos la probablidad de sufrir lesiones (RR: 1,05; 1C95\%: 1,03-1,07) y fallecidos (RR: 1,46; 1C95\%: 1,34$1,59)$ es mayor respecto de lunes a viernes.

En especial, en la franja horaria diurna de 08:00 - 19:59 (RR: 1,03; 1C95\%: 1,01-1,05) para las víctimas con lesión y nocturna de 20:00 - 07:59 (RR: 1,44; 1C95\%: 1,32-1,57) para fallecidos en A.T.

\section{Discusión}

Los resultados obtenidos son semejantes a otros estudios, dónde los A.T. siguen un patrón temporal de agrupamiento en ciertos meses, días y horas a lo largo de todo el año. ${ }^{11,12}$

Las tasas de mortalidad por A.T. según los meses del año analizados presentan variaciones cíclicas, ${ }^{13,14}$ siendo junio $(71,6)$ y octubre $(74,0)$ los meses con mayor mortalidad. No obstante, Diciembre presenta mayor ocurrencia de siniestros por A.T. (2.826), víctimas con lesión (2.128) y personas fallecidas (189) por A.T., puede ser estar influenciado al aumento del flujo vehicular por desplazamientos vacacionales en el período de navidad. ${ }^{8-10,15}$

Asimismo, a partir de los datos analizados, la distribución semanal es bastante homogénea, excepto durante el fin de semana donde se encontraron mayores tasas de morbilidad y mortalidad por A.T., en general los sábados y domingos las personas se movilizan para desarrollar actividades de ocio y diversión, implicando mayor probabilidad de sufrir un accidente y sus respectivas consecuencias. ${ }^{16} \mathrm{No}$ obstante, llama la atención que durante los días laborales los siniestros presentan estacionalidad de lunes a jueves, a excepción del día viernes que se observa un incremento porcentual del $14,9 \%$ en siniestros $(4.696 ; 15,5)$, el $20,1 \%$ en víctimas con lesión $(3.244 ; 15,2)$ y $31,4 \%$ en personas fallecidas $(290 ; 14,7)$, similar a otros estudios realizados en la población trabajadora. ${ }^{17,18}$ 
De acuerdo a la distribución por franjas horarias, los hallazgos encontrados son similares como en las de otras investigaciones, aproximadamente el $60 \%$ de los A.T. ocurren entre las 9:00 horas y las 17:00 horas, ${ }^{19}$ coincidiendo con los períodos pico del día con mayor congestión de vehicular por la entrada y salida al trabajo, ${ }^{20}$ asimismo, asociados a las víctimas con lesión. ${ }^{21}$

Se observan diferencias entre el día y la noche, la tasa de mortalidad en horario nocturno es superior al diurno. Existen multitud de factores que se añaden al componente temporal de los A.T.; circular de noche es más peligroso que durante el día por el déficit de iluminación en las vías y disminución de la visibilidad, ${ }^{22}$ exceso de velocidad ${ }^{23}$ y consumo de alcohol, ${ }^{24}$ entre otras causas.

Es importante señalar que si bien en el estudio se ha empleado el componente temporal (mes, día y hora), existen además multitud de factores causales que influyen en la morbilidad y mortalidad de los A.T., ${ }^{25}$ así como, otros métodos clásicos de análisis por conglomerados temporales. ${ }^{26,27}$ Es necesario continuar con estudios que incluyan períodos más amplios para conocer los cambios en los patrones temporales de los A.T.

A pesar de las limitaciones que presenta esta investigación, se puede considerar este estudio como el primero del país en emplear el componente temporal como factor incidente en los A.T., estos resultados permiten la comparación con investigaciones similares realizadas en otros contextos.

Además, sería interesante estudiar los accidentes de tránsito relacionados con el trabajo28. Los accidentes in-itínere han experimentado un incremento porcentual considerable en los últimos años; 5,1\% en 2013 al 20,2\% en 2016 del total de accidentes de trabajo calificados por el Seguro General de Riesgos del Trabajo del Instituto Ecuatoriano de Seguridad Social, ${ }^{28}$ fenómeno asociado al aumento de la población trabajadora afiliada y políticas sobre la notificación y prevención de accidentes de trabajo. ${ }^{29,30}$

Se considera necesario investigar esta problemática en el ámbito laboral como línea base para futuros estudios que contribuyan al campo de la seguridad laboral vial, en especial en la toma de conciencia para la prevención de este tipo de accidentes laborales. ${ }^{31}$

\section{Conclusiones}

El aporte de este estudio radica en que se identifican aquellos meses del año, días de la semana y franjas horarias con mayor concentración de los siniestros, víctimas con lesión y personas fallecidas por A.T. en el año 2016, así como, las mayores tasas de morbilidad y mortalidad según componentes temporales.

Los resultados hallados constituyen una valiosa información para establecer estrategias y campañas puntuales en cuanto al mes, día y franjas horarias, ${ }^{8}$ permitiendo optimizar la labor de los agentes de tránsito y la eficiencia de los recursos disponibles para reducir la accidentabilidad y sus conscuencias. ${ }^{32}$
Los A.T. deben continuar siendo un tema de interés prioritario para los organismos públicos, debido a los altos índices de accidentabilidad, morbilidad y mortalidad. ${ }^{3,4} \mathrm{Se}$ plantea la necesidad de reformular las políticas orientadas a la concientización social para promover la cultura de seguridad vial y peatonal, ${ }^{33}$ complementadas con la aplicación más estricta de las normas de tránsito en los meses, días y horas pico. ${ }^{34,35}$

Sería conveniente que la Agencia Nacional de Tránsito, ${ }^{9}$ como organismo encargado de registrar las estadísticas de A.T. a nivel nacional, mejorara su sistema de información pública, permitiendo a la comunidad científica realizar investigaciones más exhaustivas, aspecto ya tratado como necesario en el Informe sobre la Situación Mundial de la Seguridad Vial elaborado por la Organización Mundial de la Salud en el 2015. ${ }^{1}$

En conclusión, este estudio permite conocer la magnitud del componente temporal como factor incidente en la ocurrencia de A.T y facilitan una mejor comprensión y trascendencia de esta problemática de salud pública para el país.

\section{Fuente de Financiamiento}

Este estudio es autofinanciado

\section{Conflicto de Intereses}

No existen conflictos personales, profesionales, financieroso de otro tipo.

\section{Consentimiento Informado}

Los autores cuentan con el consentimiento informado de los paciente para la investigación, la publicación del caso y sus imágenes.

\section{Referencias Bibliográficas}

1. World Health Organization. Global status report on road safety 2015. World Health Organization; 2015.

2. Organization, Pan American Health. Status of Road Safety in the Region of the Americas. PAHO. 2019 Jun;Available from: http://iris.paho.org/xmlui/handle/ $123456789 / 51088$.

3. Algora-Buenafé AF, Russo-Puga M, SuasnavasBermúdez PR, Merino-Salazar P, Gómez-García AR. Tendencias de los accidentes de tránsito en Ecuador: 2000-2015. Revista Gerencia y Políticas de Salud. 2017;16(33):52-58.

4. Algora-Buenafé A, Suasnavas-Bermúdez P, MerinoSalazar P, Gómez-García A. Epidemiological study of fatal road traffic accidents in Ecuador. Australasian Medical Journal (Online). 2017;10(3):238.

5. Galarza-Velasteguí LA, Merino-Salazar P, AlgoraBuenafé AF, Gómez-García AR. Estudio geoespacial de los accidentes de tránsito en la Región Amazónica Ecuatoriana. Revista Cienciamérica. 2017;6(2):31-36. 
6. Algora-Buenafé FA, Claudio T, Oscar M, Gómez-García 19. Kar S, Das S, Tiwari A, Pharveen I. Pattern of road traffic AR. Análisis espacial de los accidentes de tránsito en los Cantones de la Provincia de Pichincha, 2016. 2017; accidents in Bhubaneswar, Odisha. Clinical epidemiology and global health. 2016;4(3):115-119.

7. Gallegos AdPP, Portalanza A, Samaniego CEE, Salazar 20. del Pilar Camacho M. Caracterización espacio-temporal PM, García ARG. Mortalidad y Años de Vida Potencialmente Perdidos por Accidentes de Tránsito en Ecuador. CienciAmérica: Revista de divulgación científica de la Universidad Tecnológica Indoamérica. 2018;7(1):11-21. y determinación de los patrones territoriales de alta concentración de la accidentalidad en la vía Tunja-Moniquirá. Perspectiva Geográfica: Revista del Programa de Estudios de Posgrado en Geografía. 2010;(15):225-238.

8. Piña-Tornés A, González-Longoria L, González-Pardo S, 21. Plug C, Xia JC, Caulfield C. Spatial and temporal visualiAcosta-González A, Vintimilla-Burgos P, Paspuel-Yar S. Mortalidad por accidentes de tránsito en Bayamo, Cuba 2011. Revista Peruana de Medicina Experimental y Salud 22. Pública. 2014;31:721-724.

9. Agencia Nacional de Tránsito. Estadísticas de transporte terrestre y seguridad vial, Ecuador; 2016. Available from: https://www.ecuadorencifras.gob.ec/documentos/webinec/Estadisticas_Economicas/Estadistica\%20de\% 20Transporte/2016/2016_AnuarioTransportes_ Resumen\%20Metodol\%C3\%B3gico.pdf. sation techniques for crash analysis. Accident Analysis \& Prevention. 2011;43(6):1937-1946.

2. Cardona-Arbeláez SA, Molina-Castaño CF, Arangoálzate CM, Pichott-Padilla JT. Caracterización de accidentes de tránsito y valoración tarifaria de la atención médica en el servicio de urgencias, Caldas-Antioquia 2007-2008. Gerencia y Políticas de Salud. 2010;9(19).

23. Whitelegg J. A geography of road traffic accidents. Transactions of the Institute of British Geographers. 1987;p. 161-176.

10. Farchi S, Molino N, Rossi PG, Borgia P, Krzyzanowski M, 24. Shahid S, Minhans A, Puan OC, Hasan SA, Ismail T. SpaDalbokova D, et al. Defining a common set of indicators to monitor road accidents in the European Union. BMC Public Health. 2006;6(1):183.

11. Vargas WE, Mozo E, Herrera E, et al. Análisis de los puntos más críticos de accidentes de tránsito en Bogotá. Revista De TopografíA Azimut. 2012;4.

12. Jorge Miguez A, Godoy del Sol H, Ortis Sagasta M. Caracterización de la mortalidad por accidentes del tránsito 26. con participación de ciclos: un problema sociomédico. MediSur. 2010;8(4):57-62.

13. Ruiz JI, Herrera AN. Accidentes de tránsito con heridos en Colombia según fuentes de información: caracteriza- 27. ción gene-ral y tipologías de accidentes. Ces Psicología. 2016;9(1):32-46.

14. Cardona AMS, Arango DC, FernándezI DYB, Martínez AA. Mortalidad por accidente de tránsito en el adulto mayor en Colombia. Revista de Saúde Pública. 2017;51:1-8.

15. Cervantes-Trejo A, Leenen I, Fabila-Carrasco JS, Rojas- 29 Vargas R. Trends in traffic fatalities in mexico: examining progress on the decade of action for road safety 2011-2020. International journal of public health. 2016;61(8):903-913.

16. Choquehuanca-Vilca V, Cárdenas-García F, CollazosCarhuay J, Mendoza-Valladolid W. Perfil epidemiológico de los accidentes de tránsito en el Perú, 2005-2009. Revista Peruana de Medicina Experimental y Salud Pública. 2010;27:162-169.

17. Reinoso-Barbero L, Díaz-Garrido R, González-Gómez MF, Fernández-Fernández M, Capapé-Aguilar A, Garrido-Astray MC. Lesiones por accidentes de trabajo 32. Mohan VR, Sarkar R, Abraham VJ, Balraj V, Naumova relacionados con el tráfico ocurridas en los trabajadores de una entidad bancaria (2007-2013). Archivos de Prevención de Riesgos Laborales. 2015;18(4):185-191.

18. Cruz Toscano VA, Barrios Queipo EA, Gallar Pérez Y, tial and temporal pattern of road accidents and casualties in Peninsular Malaysia. Jurnal Teknologi. 2015;14:57-65.

25. Hidalgo-Solórzano EdC, Híjar M, Blanco-Muñoz J, Kageyama-Escobar MdlL. Factores asociados con la gravedad de lesiones ocurridas en la vía pública en Cuernavaca, Morelos, México. salud pública de méxico. 2005;47:30-38.

6. Mantel N, Kryscio RJ, Myers MH. Tables and formulas for extended use of the Ederer-Myers-Mantel diseaseclustering procedure. American Journal of Epidemiology. 1976;104(5):576-584.

27. Grimson RC. Disease clusters, maxima, and P-values. Statistics in Medicine. 1993;12(19-20):1773-1794.

28. Instituto Ecuatoriano de Seguridad Social. Estadísticas del Seguro de Riesgos del Trabajo- Seguro general de Riesgos de Trabajo; 2016. Available from: http://sart.iess.gob.ec/ SRGP/indicadores_ecuador.php.

9. Gómez García AR, Algora Buenafé AF, Bermúdez S, Roberto P, Vilaret Serpa A, et al. Notificación de accidentes de trabajo y posibles enfermedades profesionales en Ecuador, 2010-2015. Ciencia \& trabajo. 2016;18(57):166-172.

30. Gómez García AR, Merino Salazar P, Tapia Claudio OM, Espinoza Samaniego CE, Echeverría López MA. Epidemiología de accidentes de trabajo en Ecuador basado en la base de datos de la Seguridad Social en los años 20142016. Revista SCientifica. 2017;15:14.

31. de Vicente MA, Verdejo MZ, de la Orden Rivera MV. El accidente laboral de tráfico. Archivos de prevención de riesgos laborales. 2012;15(1):5-6.

EN. Differential patterns, trends and hotspots of road traffic injuries on different road networks in Vellore district, southern India. Tropical Medicine \& International Health. 2015;20(3):293-303.

Gómez-García AR. Risk of in-itinere accident in primary 33. Merchán MEP, Pérez REG, Aristizábal OPN. Seguridad health care professionals. 2016;. vial y peatonal: una aproximación teórica desde la polí- 
tica pública. Revista Hacia la Promoción de la Salud. 2011;16(2):190-204.

34. Zangeneh A, Najafi F, Karimi S, Saeidi S, Izadi N. Spatialtemporal cluster analysis of mortality from road traffic injuries using geographic information systems in West of Iran during 2009-2014. Journal of forensic and legal medicine. 2018;55:15-22.

35. Padilla JIY. Lesiones físicas en accidentes de tránsito, en el cantón Cañar. Killkana Salud y Bienestar. 2017;1(1):11-14.

Recibido: 16 de marzo de 2018

Aceptado: 12 de febrero de 2019 
